\title{
Unendlich oft teilbare \\ Wahrscheinlichkeitsverteilungen auf kompakten Gruppen
}

\section{Doctoral Thesis}

Author(s):

Carnal, Henri

Publication date:

1963

Permanent link:

https://doi.org/10.3929/ethz-a-000087900

Rights / license:

In Copyright - Non-Commercial Use Permitted 
Prom. Nr. 3375

\title{
Unendlich oft teilbare Wahrscheinlichkeitsverteilungen auf kompakten Gruppen
}

\author{
Von der \\ Eidgenössischen Technischen \\ Hochschule in Zürich \\ zur Erlangung \\ der Würde eines Doktors der Mathematik \\ genehmigte \\ Promotionsarbeit
}

Vorgelegt von

Henri Carnal

dipl. Math.

von Souboz (Bern)

Referent: Herr Prof. Dr. B. Eckmann

Korreferent: Herr Prof. Dr. W. Saxer

Zürich 1963

Druck der Brühlschen Universitätsdruckerei Gießen 


\title{
Unendlich oft teilbare Wahrscheinlichkeitsverteilungen auf kompakten Gruppen
}

\author{
Von
}

\section{Henri Carnal in Zürich}

Viele Arbeiten über Verteilungen auf topologischen lokalkompakten Gruppen beschränken sich auf den speziellen Fall, wo die Gruppe kommutativ ist. In dieser Abhandlung werden einige Sätze, die zuerst nur im abelschen Fall bewiesen wurden, auf allgemeine kompakte Gruppen übertragen.

Nach einigen Vorbereitungen in Kap. I wird in Kap. II ein Satz von URBANIK [13] über Poissonverteilungen für beliebige kompakte Gruppen bewiesen, in Kap. III einige Sätze von KLoss [6] über die Einbettung unendlich oft teilbarer Verteilungen in stetige Halbgruppen, speziell auf Lieschen und total unzusammenhängenden Gruppen (Satz 3, 5, 6, 7) und in Kap. IV ein Satz von URBANIK [14] über die Existenz Gaußscher Verteilungen auf zusammenhängenden Gruppen (Satz 8) sowie zwei Sätze von BocHNER [1] über untergeordnete Halbgruppen (Satz 12 und 13). Weiter wird der Satz von Hrule-Yosida (vgl. [9], § I.4) für den speziellen Fall von Wahrscheinlichkeitshalbgruppen auf kompakten Gruppen in vereinfachter Form gegeben (Satz 4). Satz 2 gibt eine Möglichkeit, alle unendlich oft teilbaren Verteilungen aus den stetigen Halbgruppen zu gewinnen; sie wird später in Satz 10 bei der Beschreibung der Gaußschen Verteilungen benützt, nachdem in Satz 9 die stetigen Halbgruppen Gaußscher Verteilungen charakterisiert worden sind. Schließlich wird in Satz 11 eine wichtige Klasse symmetrischer Halbgruppen beschrieben.

Diese Arbeit entstand während eines einjährigen Aufenthaltes an der Universität Wien, der von einem Stipendium des "Rotary International" finanziert wurde. Es ist mir eine besonders angenehme Pflicht, mich bei Herrn Prof. Scmmetterer zu bedanken, der die Anregung zu dieser Arbeit gab und mir stets mit wertvollen Ratschlägen zur Seite stand.

\section{Hilfsmittel, Bezeichnungen}

Sei $G$ eine kompakte topologische Gruppe, $L$ die Banachalgebra der stetigen Funktionen auf $G$ mit der Norm $\|f\|=\sup _{x \in G}|f(x)|, R$ die Menge der Radonschen Masse auf $G$. Jedem $\mu \in R$ entspricht ein lineares Funktional $\varphi_{\mu}$ auf $L$ :

$$
\varphi_{\mu}(f)=\int_{G} f(x) d \mu(x)
$$

und umgekehrt. Das Faltungsprodukt $\mu \nu$ kann man entweder durch

$$
\mu \nu(E)=\int_{G} \mu\left(E x^{-1}\right) d \nu(x)
$$

für die Borelschen Mengen $E(G$ oder durch

$$
\varphi_{\mu \nu}(f)=\int_{G} \int_{G} f(x y) d \mu(x) d \nu(y)
$$

